PROCEEDINGS OF THE

AMERICAN MATHEMATICAL SOCIETY

Volume 128, Number 2, Pages 361-367

S 0002-9939(99)04996-5

Article electronically published on July 6, 1999

\title{
ON POINTED HOPF ALGEBRAS OF DIMENSION $p^{n}$
}

\author{
M. BEATTIE, S. DĂSCĂLESCU, AND L. GRÜNENFELDER \\ (Communicated by Ken Goodearl)
}

\begin{abstract}
In this note we describe nonsemisimple Hopf algebras of dimension $p^{n}$ with coradical isomorphic to $k C, C$ abelian of order $p^{n-1}$, over an algebraically closed field $k$ of characteristic zero. If $C$ is cyclic or $C=\left(C_{p}\right)^{n-1}$, then we also determine the number of isomorphism classes of such Hopf algebras.
\end{abstract}

\section{INTRODUCTION AND PRELIMINARIES}

In recent years considerable effort has been made to classify finite dimensional Hopf algebras over an algebraically closed field $k$ of characteristic 0 . In [17], Zhu proved that a Hopf algebra of prime dimension $p$ is isomorphic to $k C_{p}$. For the semisimple case, a series of results has appeared. Masuoka has classified semisimple Hopf algebras of dimensions $6,8, p^{2}, p^{3}$ and $2 p$ for $p$ an odd prime (see [8], [9], [10], [11]). Larson and Radford [7] showed that a semisimple Hopf algebra of dimension $\leq 19$ is commutative and cocommutative, and thus isomorphic to a group algebra. Also, in [4], Gelaki constructs interesting examples of semisimple Hopf algebras of dimension $p q^{2}, p, q$ distinct primes. The nonsemisimple case seems to be more difficult. In dimension $p^{2}$, apart from $k C_{p^{2}}$ and $k\left(C_{p} \times C_{p}\right)$ which are semisimple, $p-1$ types of nonsemisimple Hopf algebras are known. These are the Taft Hopf algebras, which we denote by $T_{\lambda}, \lambda$ a primitive $p$ th root of 1 . The algebras $T_{\lambda}$ are pointed with coradical $k C_{p}$. Conversely, if $H$ is a pointed nonsemisimple Hopf algebra of dimension $p^{2}, H$ is a Taft Hopf algebra.

Here, we describe Hopf algebras of dimension $p^{n}, n \geq 3$, which have coradical $k C, C$ abelian of order $p^{n-1}$, and note that the situation is somewhat different from the dimension $p^{2}$ case. The Hopf algebras which occur can be obtained by an Ore extension construction as in [2] or [3]; if $C$ is cyclic, there are $p^{\left[\frac{n}{2}\right]}+p^{\left[\frac{n-1}{2}\right]}+p-3$ nonisomorphic such Hopf algebras; if $C=\left(C_{p}\right)^{n-1}$, then there are $p-1$ isomorphism classes.

Throughout, $k$ is an algebraically closed field of characteristic 0 . We follow the standard notation in [12]. For $H$ a Hopf algebra, $G(H)$ will denote the group of grouplike elements and $H_{0}, H_{1}, H_{2}, \ldots$ will denote the coradical filtration of $H$. $H$ is called pointed if $H_{0}=k G(H)$. If $g, h \in G(H)$ then the set of $(g, h)$-primitive elements is $P_{g, h}=\{x \in H \mid \triangle(x)=x \otimes g+h \otimes x\}$. Since $g-h \in P_{g, h}$ we can choose a subspace $P_{g, h}^{\prime}$ of $P_{g, h}$ such that $P_{g, h}=k(g-h) \oplus P_{g, h}^{\prime}$. We will need the

Received by the editors October 7, 1997 and, in revised form, April 3, 1998.

1991 Mathematics Subject Classification. Primary 16W30.

The first and third authors research was partially supported by NSERC.

(C)1999 American Mathematical Society 
version of the Taft-Wilson theorem proved in [12, Theorem 5.4.1], which states that $H_{1}=H_{0} \oplus\left(\bigoplus_{g, h \in G(H)} P_{g, h}^{\prime}\right)$ for a finite dimensional pointed Hopf algebra $H$. If $H$ is finite dimensional, $P_{1,1}=0$. This implies that if $H$ is also pointed of dimension $>1$, then $G(H)$ is not trivial.

The notation $\left(\begin{array}{l}t \\ j\end{array}\right)_{q}$ denotes a $q$-binomial coefficient. Details on the definitions of these coefficients can be found in any introduction to quantum groups, for example, [6, IV.2] or the introductory section of [15].

An efficient method for constructing nonsemisimple finite dimensional pointed Hopf algebras by means of iterated Ore extensions was developed in [3]. If a single Ore extension of the group algebra of a finitely generated abelian group $C$ is involved, then, simply put, the method consists of taking an automorphism $\varphi$ of the group algebra $k C$ of the form $\varphi(c)=\lambda^{-1} c, \lambda \in k$, for each generator $c$ of $C$, and forming the Ore extension $k C[X, \varphi]$. Comultiplication is defined on $X$ by $\triangle(X)=g \otimes X+X \otimes 1$, for some $g \in C$. This defines a Hopf algebra structure on the infinite dimensional space $k C[X, \varphi]$. Then we factor out by a Hopf ideal of finite codimension. For more details about the general construction, see $[2, \S 4]$ or [3]. The specific Hopf algebras used in this note are described below.

\section{The Classification Results}

Before proving our results, we define Hopf algebras $H(\lambda, u)$ and $\tilde{H}(\lambda, u)$ of dimension $p^{n}$. These Hopf algebras are exactly Ore extension constructions as in $[2$, $\S 4]$ or [3].

Fix a prime $p$ and an integer $n \geq 2$. Let $C=C_{1} \times \ldots \times C_{m}$ be a finite abelian group of order $p^{n-1}$, where $C_{i}=\left\langle c_{i}\right\rangle$ is cyclic of order $p^{n_{i}}$, and let $\phi$ be an automorphism of $k C$ defined by $\phi\left(c_{i}\right)=\lambda_{i}^{-1} c_{i}$. Then clearly $\lambda_{i}^{p^{n_{i}}}=1$. We write $c^{u}$ to denote $c_{1}^{u_{1}} \ldots c_{m}^{u_{m}}$ and $\lambda^{u}$ to denote $\lambda_{1}^{u_{1}} \ldots \lambda_{m}^{u_{m}}$ if $u=\left(u_{1}, \ldots, u_{m}\right)$ is an $m$-tuple of integers. As outlined in the introduction, we define a Hopf algebra structure on the Ore extension $k C[X, \phi]$ by setting $\Delta(X)=c^{u} \otimes X+X \otimes 1$ for some element $c^{u} \neq 1$ of $C$, and $\epsilon(X)=0$. The Hopf algebra $k C[X, \phi]$ has generators $c_{i}$, $1 \leq i \leq m$, and $X$, relations

$$
c_{i}^{p^{n_{i}}}=1, \quad X c_{i}=\lambda_{i}^{-1} c_{i} X
$$

and coalgebra structure induced by

$$
\triangle\left(c_{i}\right)=c_{i} \otimes c_{i}, \quad \triangle(X)=c^{u} \otimes X+X \otimes 1, \quad \epsilon\left(c_{i}\right)=1, \quad \epsilon(X)=0,
$$

where $u=\left(u_{1}, u_{2}, \ldots, u_{m}\right) \in \mathbf{Z}^{m}$ and $c^{u}=c_{1}^{u_{1}} c_{2}^{u_{2}} \ldots c_{m}^{u_{m}}$. The antipode is given by $S\left(c_{i}\right)=c_{i}^{-1}, S(X)=-c^{-u} X$. Then $k C[X, \phi]$ is an infinite dimensional pointed Hopf algebra with $G(k C[X, \phi])=C$.

Now let $\lambda^{u}=\lambda_{1}^{u_{1}} \ldots \lambda_{m}^{u_{m}}$ be a primitive $p$ th root of unity. Write $c^{u p}$ for $\left(c^{u}\right)^{p}=$ $c_{1}^{u_{1} p} \ldots c_{m}^{u_{m} p}$. Then $\Delta\left(X^{p}\right)=c^{u p} \otimes X^{p}+X^{p} \otimes 1$, so that the ideal generated by $X^{p}$ is a Hopf ideal in $k C[X, \phi]$. If $\lambda^{u}$ is a primitive $p$ th root of unity and if also $c^{u}$ has order different from $p$, the ideal generated by $X^{p}-c^{u p}+1$ is a different Hopf ideal. The quotient Hopf algebras

$$
H(\lambda, u)=k C[X, \phi] /\left\langle X^{p}\right\rangle, \quad \tilde{H}(\lambda, u)=k C[X, \phi] /\left\langle X^{p}-c^{u p}+1\right\rangle
$$

are pointed Hopf algebras of dimension $p^{n}$ and $G(H(\lambda, u)) \cong C \cong G(\tilde{H}(\lambda, u))[14$, Lemma 1]. Let $x$ denote the coset of $X$.

Note that in both cases $P_{1, g}^{\prime} \neq 0$ only if $g=c^{u}$ and that $\operatorname{dim} P_{1, c^{u}}^{\prime}=1$. We see below that the cases $H$ and $\tilde{H}$ are distinct. 
Lemma 1. For $C$ an abelian group of order $p^{n-1}$ and $H(\lambda, u), \tilde{H}(\xi, v)$ as above, then $H(\lambda, u)$ cannot be isomorphic to $\tilde{H}(\xi, v)$.

Proof. Suppose $\phi: \tilde{H}(\xi, v) \rightarrow H(\lambda, u)$ is a Hopf algebra isomorphism. Then $\phi$ induces an automorphism of the group $C$. Now $\operatorname{dim} P_{1, g}=2$ in $\tilde{H}(\xi, v)$ if and only if $\operatorname{dim} P_{1, \phi(g)}=2$ in $H(\lambda, u)$. So $\phi\left(c^{v}\right)=c^{u}$ and $0 \neq x \in P_{1, c^{v}}-k\left(c^{v}-1\right) \subset \tilde{H}(\xi, v)$, so that $\phi(x)=\alpha\left(\phi\left(c^{v}\right)-1\right)+y$ for some $\alpha \in k$ and some $0 \neq y \in P_{1, c^{u}}-k\left(c^{u}-1\right) \subset$ $H(\lambda, u)$ with $y^{p}=0$.

Since $\phi\left(x c^{v}\right)=\phi(x) \phi\left(c^{v}\right)=\phi\left(\xi^{-v} c^{v} x\right)=\xi^{-v} \phi\left(c^{v}\right) \phi(x)$, we see that $\alpha=0$, and $\phi(x)=y$. But then $x^{p}=0$, in contradiction to the definition of $\tilde{H}(\xi, v)$.

If $C=C_{p} \times \ldots \times C_{p}=C_{p}^{n-1}$ then no $\tilde{H}(\lambda, u)$ occur, since every element of $C$ has order $p$. In particular, when $n=2$ then $C=C_{p}$, and the Hopf algebras we get are isomorphic to the Taft Hopf algebras of dimension $p^{2}$, denoted $T_{\lambda}, \lambda$ a primitive $p$ th root of 1 . There are $p-1$ nonisomorphic $T_{\lambda}$.

In $\tilde{H}(\lambda, u)$, if $C=C_{p^{n-1}}$, then $x^{p}=c^{u p}-1$ commutes with $c$, so that $\lambda^{p}=1$. Thus $\lambda$ is a primitive $p$ th root of unity, and since $\lambda^{u}$ is also a primitive $p$ th root, $p$ does not divide $u$ and $c^{u}$ is a generator of $C$.

We can now describe Hopf algebras of dimension $p^{n}$ with coradical $k C$, where $C$ is an abelian group of order $p^{n-1}$.

Theorem 2. A Hopf algebra $H$ of dimension $p^{n}$ whose coradical $H_{0}$ is the group algebra of an abelian group of order $p^{n-1}$ is isomorphic to $H(\lambda, u)$ or $\tilde{H}(\lambda, u)$ for some $(\lambda, u)$.

Proof. Let $C=\times_{i=1}^{m} C_{i}$ be a cyclic decomposition with generators $c_{1}, c_{2}, \ldots, c_{m}$. The Taft-Wilson theorem says that $H_{1}=H_{0} \oplus\left(\oplus_{g, h \in C} P_{g, h}^{\prime}\right)$. Observe that $a P_{g, h} b=$ $P_{a g b, a h b}$ for any $a, b \in C$. Consider the action of $C$ on $H_{1}$ given by conjugation, i.e. for each $g \in C$ the automorphism $T_{g}: H_{1} \rightarrow H_{1}$ is defined by $T_{g}(y)=g y g^{-1}$. Then $T_{g}^{p^{n-1}}$ is the identity and the eigenvalues of $T_{g}$ satisfy the equation $\lambda^{p^{n-1}}=1$. Since $C$ is abelian, each $P_{g, h}$ is invariant under this action and has a basis of joint eigenvectors, i.e. vectors $w$ such that $T_{a}(w)=a w a^{-1}=\lambda_{a} w$ for every $a \in C$. Since $H$ is not commutative there is a nonzero joint eigenvector $w \notin H_{0}$ contained in some $P_{g, h}$ and in fact in $P_{1, r}$ for some $1 \neq r=c_{1}^{s_{1}} c_{2}^{s_{2}} \ldots c_{m}^{s_{m}}=c^{s}, s=\left(s_{1}, \ldots, s_{m}\right) \in \mathbf{Z}^{m}$, whose eigenvalue $\lambda_{i} \neq 1$ for at least one generator $c_{i}$. Thus, we have

$$
\Delta(w)=r \otimes w+w \otimes 1, \quad c_{i} w=\lambda_{i} w c_{i} .
$$

The Hopf subalgebra of $H$ generated by $\left\{c_{1}, c_{2}, \ldots, c_{m}, w\right\}$ is the subspace spanned by the elements $c^{i} w^{j}$ with $i \in \mathbf{N}^{m}$ and $j \in \mathbf{N}$. By the Nichols-Zoeller Theorem $[12,3.1 .5]$ this Hopf subalgebra must be all of $H$. Note that $(w \otimes 1)(r \otimes w)=$ $\lambda^{-s}(r \otimes w)(w \otimes 1)$. Also $\lambda^{-s} \neq 1$ since otherwise $r$ and $w$ commute and so generate a commutative nonsemisimple Hopf subalgebra of $H$, which then must be infinite dimensional. Thus $\lambda^{-s}$ is a primitive root of unity of order $p^{l}$ for some $l>0$, so that for $t \leq p^{l}$

$$
\Delta\left(w^{t}\right)=\sum_{j=0}^{t}\left(\begin{array}{l}
t \\
j
\end{array}\right)_{\lambda^{-s}} r^{t-j} w^{j} \otimes w^{t-j} .
$$

We prove by induction on $t, 1 \leq t \leq p-1$, that $w^{t} \in H_{t}-H_{t-1}$. For $t=1$ the statement is obvious. Assume that $w^{v} \in H_{v}-H_{v-1}$ for $v<t$. Then $r^{t} \otimes w^{t} \in$ $H_{0} \otimes H, r^{t-j} w^{j} \otimes w^{t-j} \in H \otimes H_{t-2}$ for $j \geq 2$, and $r^{t-1} w \otimes w^{t-1} \in H \otimes H_{t-1}$. 
Thus, $\Delta\left(w^{t}\right) \in H_{0} \otimes H+H \otimes H_{t-1}$, and it follows that $w^{t} \in H_{t}$. If $w^{t} \in H_{t-1}$, then $\Delta\left(w^{t}\right) \in H_{0} \otimes H+H \otimes H_{t-2}$; hence $\left(\begin{array}{l}t \\ 1\end{array}\right)_{\lambda^{-s}} r^{t-1} w \otimes w^{t-1} \in H_{0} \otimes H+H \otimes H_{t-2}$. Since $t<p$, we must have $\left(\begin{array}{l}t \\ 1\end{array}\right)_{\lambda^{-s}} \neq 0$. Now $r^{t-1} w \in H_{1}-H_{0}$ and $w^{t-1} \notin H_{t-2}$ gives a contradiction, and thus $w^{t} \in H_{t}-H_{t-1}$.

By [14, Corollary 2.3], $H_{t}$ is a free $H_{0}$-module so that $\operatorname{dim} H_{t} \geq(t+1) p^{n-1}$ for $0 \leq t \leq p-1$ and in particular $\operatorname{dim} H_{p-1} \geq p^{n}$. This shows that $H=H_{p-1}$ and $\operatorname{dim} H_{t}=(t+1) p^{n-1}$ for $t \leq p-1$. Thus, $\operatorname{dim} H_{1}=2 p^{n-1}$ and by the Taft-Wilson Theorem the subset $\{h w \mid h \in C\}$ of $H_{1}-H_{0}$ is linearly independent in $H_{1}$, so that $\operatorname{dim} P_{h, h r}=2$ for all $h \in C$.

We claim that the subset $\left\{h w^{j} \mid h \in C, 0 \leq j \leq p-1\right\}$ of $H$ is linearly independent. If not, then there is some $t \leq p-1$ such that

$$
\sum_{h \in C} \alpha_{h} h w^{t}=\sum_{h \in C, 0 \leq q<t} \beta_{h, q} h w^{q}
$$

for some scalars $\alpha_{h}$ and $\beta_{h, q}$ with $\alpha_{g} \neq 0$ for some $g \in G$. The image of the comultiplication map $\Delta$ applied to the right hand side of the equation above lies in $H \otimes H_{t-1}$. Let $\psi \in H^{*}$ be such that $\psi\left(H_{t-1}\right)=0$ and $\psi\left(g w^{t}\right)=1$, and also let $\phi \in H^{*}$ be such that $\phi\left(g r^{t}\right)=1$ and $\phi\left(h r^{t}\right)=0$ for $h \neq g$. Then $(\phi \otimes \psi)\left(H \otimes H_{t-1}\right)=0$ but $\phi \otimes \psi$ applied to the left hand side of the equation above yields $(\phi \otimes \psi)\left(\sum_{h \in C} \alpha_{h} h r^{t} \otimes h w^{t}\right)=\alpha_{g} \neq 0$, a contradiction.

Since $H_{p-1}=H$, we have that $w^{p} \in H_{p-1}$, so that $\Delta\left(w^{p}\right) \in H_{0} \otimes H+H \otimes H_{p-2}$. Expanding as above, we find that $\left(\begin{array}{l}p \\ 1\end{array}\right)_{\lambda^{-s}} r^{p-1} w \otimes w^{p-1} \in H_{0} \otimes H+H \otimes H_{p-2}$. Since $r^{p-1} w \in H_{1}-H_{0}$ and $w^{p-1} \notin H_{p-2}$ we conclude that $\left(\begin{array}{l}p \\ 1\end{array}\right)_{\lambda^{-s}}=0$, so that $\lambda^{-s}$ is a primitive $p$ th root of 1 . Then $\left(\begin{array}{l}p \\ j\end{array}\right)_{\lambda^{-s}}=0$ for $1 \leq j \leq p-1$ and

$$
\Delta\left(w^{p}\right)=r^{p} \otimes w^{p}+w^{p} \otimes 1
$$

so that $w^{p} \in P_{1, r^{p}}=k\left(r^{p}-1\right) \oplus P_{1, r^{p}}^{\prime}$. Since $r^{p} \neq r$ we have $P_{1, r^{p}}=k\left(r^{p}-1\right)$ and $w^{p}=\gamma\left(r^{p}-1\right)$ for some scalar $\gamma$.

If $w^{p}=0$, then it is clear that $H \cong H(\lambda, s)$. If $w^{p} \neq 0$, then replacing $w$ by $\gamma^{-1 / p} w$, we see that $H \cong \tilde{H}(\lambda, s)$.

Corollary 3. The only nonsemisimple pointed Hopf algebras of dimension $p^{2}$ are the Taft-Hopf algebras.

Now we show that if $C=\left(C_{p}\right)^{n-1}=\left\langle c_{1}\right\rangle \times\left\langle c_{2}\right\rangle \times \ldots \times\left\langle c_{n-1}\right\rangle$, then a result similar to Corollary 3 holds.

Proposition 4. If $C=\left(C_{p}\right)^{n-1}$, then $H(\lambda, u) \cong k\left(C_{p}\right)^{n-2} \otimes T_{\lambda_{i}}$ for some $i$, and there are exactly $p-1$ isomorphism classes of such Hopf algebras.

Proof. We argue by induction on $n$. If $n-1=1$, then the result follows from Corollary 3. Recall that $c^{u} \neq 1$, since $\operatorname{dim} P_{1,1}=0$ and also $\lambda^{u} \neq 1$, else $c^{u}$ and $x$ commute and $H(\lambda, u)$ could not be finite dimensional. Thus some $\lambda_{i}^{u_{i}} \neq 1$, and we may assume that $\lambda_{2}^{u_{2}} \neq 1$, in particular $\lambda_{2} \neq 1$ and $u_{2} \neq 0$. Now we distinguish two cases.

If $\lambda_{1}=1$, then let $u^{\prime}=\left(u_{2}, u_{3}, \ldots, u_{n-1}\right)$ and $\lambda^{\prime}=\left(\lambda_{2}, \lambda_{3}, \ldots, \lambda_{n-1}\right)$. Then the map

$$
f: H(\lambda, u) \rightarrow H\left(\left(1, \lambda^{\prime}\right),\left(0, u^{\prime}\right)\right) \cong k C_{p} \otimes H\left(\lambda^{\prime}, u^{\prime}\right)
$$


defined by

$$
f(x)=x, \quad f\left(c_{1}\right)=c_{1}^{-u_{2}}, \quad f\left(c_{2}\right)=c_{1}^{u_{1}} c_{2}, \quad f\left(c_{i}\right)=c_{i}
$$

for $i>2$ is an isomorphism of Hopf algebras.

If $\lambda_{1} \neq 1$, then $\lambda_{1}=\lambda_{2}^{h}$ for some $h$. Now let $u^{\prime}=\left(h u_{1}+u_{2}, u_{3}, \ldots, u_{n-1}\right)$ and $\lambda^{\prime}=\left(\lambda_{2}, \lambda_{3}, \ldots, \lambda_{n-1}\right)$. Then the map

$$
f: H(\lambda, u) \rightarrow H\left(\left(1, \lambda^{\prime}\right),\left(0, u^{\prime}\right)\right) \cong k C_{p} \otimes H\left(\lambda^{\prime}, u^{\prime}\right)
$$

defined by

$$
f(x)=x, \quad f\left(c_{1}\right)=c_{1}^{h u_{2}} c_{2}^{h}, \quad f\left(c_{2}\right)=c_{1}^{-h u_{1}} c_{2}, \quad f\left(c_{i}\right)=c_{i}
$$

for $i>2$ is an isomorphism.

Thus, if the assertion holds for $C=\left(C_{p}\right)^{n-2}$, it holds for $C=\left(C_{p}\right)^{n-1}$ and we are done.

Finally, we count the number of isomorphism classes of Hopf algebras of dimension $p^{n}$ with coradical $k C_{p^{n-1}}$. First we need two lemmas. From now on, since $C=\langle c\rangle$ is cyclic, the notation $H(\lambda, i)$ refers to $\lambda \in k, i \in \mathbf{Z}$. Also, since in the cyclic case in $\tilde{H}(\lambda, u)$ the element $c^{u}$ is a generator of $C$ we may assume that $u=1$, and we abbreviate $\tilde{H}(\lambda, 1)$ by $H(\lambda)$.

Lemma 5. $H(\lambda, i) \simeq H(\mu, j)$ if and only if there exists $h$ relatively prime to $p$ such that $\lambda=\mu^{h}$ and $j \equiv h i\left(\bmod p^{n-1}\right)$.

Proof. Let $f: H(\lambda, i) \rightarrow H(\mu, j)$ be a Hopf algebra isomorphism. Then $f$ induces an automorphism of the coradical $k C_{p^{n-1}}$, so that $f(c)=c^{h}$ for some $h$ relatively prime to $p$.

Furthermore, let $x \in P_{c^{i}, 1}^{\prime}$ in $H(\lambda, i)$. Then $P_{c^{h i}, 1}$ in $H(\mu, j)$ is nonzero, so $c^{h i}=c^{j}$. Thus $h i \equiv j \bmod p^{n-1}$. Finally, since $c x=\lambda x c, c^{h} f(x)=\lambda f(x) c^{h}$. But $f(x) \in P_{c^{j}, 1}$ and the above equation implies that $f(x) \in P_{c^{j}, 1}^{\prime}$ so that $c f(x)=$ $\mu f(x) c$ in $H(\mu, j)$. Then $c^{h} f(x)=\mu^{h} f(x) c^{h}$, so $\lambda=\mu^{h}$.

Conversely, given such an integer $h$, define $f$ by $f(c)=c^{h}$ and $f(x)=z \in$ $P_{g^{j}, 1}^{\prime}$.

Lemma 6. $H(\lambda) \simeq H(\mu)$ if and only if $\lambda=\mu$. Thus there are exactly $p-1$ nonisomorphic $H(\lambda)$.

Proof. As in Lemma 5, if $f: H(\lambda) \rightarrow H(\mu)$ is a Hopf algebra isomorphism, $f(c)=$ $c^{h}$ for some $h$. Let $x \in P_{1, c}^{\prime}$ in $H(\lambda)$. Then $f(x) \in P_{1, c^{h}}$ in $H(\mu)$ which implies that $h=1$. The result then follows immediately.

Proposition 7. For $n \geq 3$, there exist precisely $p^{\left[\frac{n}{2}\right]}+p^{\left[\frac{n-1}{2}\right]}+p-3$ Hopf algebras of dimension $p^{n}$ with coradical $k C_{p^{n-1}}$, where $[y]$ is the largest integer less than or equal to $y$.

Proof. We first count the Hopf algebras of the form $H(\lambda, i)$. If $H(\lambda, i) \simeq H(\mu, j)$, then $\lambda$ and $\mu$ must have the same order. Let us fix the order of $\lambda$, say $p^{b}, 1 \leq b \leq$ $n-1$. Then by Lemma 5 , we can fix $\lambda$. Since $\lambda^{i}$ is primitive of order $p, i=q p^{b-1}$ where $(p, q)=1$ and $1 \leq q \leq p^{n-b}$. Thus there are $p^{n-b}-p^{n-b-1}$ choices for $i$. For a fixed such $i$ we have $H(\lambda, i) \simeq H(\lambda, j)$ if and only if there exists an $h$ not divisible by $p$ with $\lambda^{h}=\lambda$ and $j \equiv h i\left(\bmod p^{n-1}\right)$. But $\lambda^{h}=\lambda$ implies that $h=\alpha p^{b}+1$, 
$0 \leq \alpha \leq p^{c-b}-1$. Since for $h=\alpha p^{b}+1, h^{\prime}=\beta p^{b}+1$ we have $h i \equiv h^{\prime} i\left(\bmod p^{n-1}\right)$ if and only if $p^{n-b} \mid(\alpha-\beta) p^{b}$, we distinguish two cases. If $b \geq \frac{n}{2}$, then $h i \equiv i(\bmod$ $p^{n-1}$ ) for all the indicated $h$ 's, and in this case $H(\lambda, i) \simeq H(\lambda, j)$ implies $i=j$. Therefore there exist $p^{n-b}-p^{n-b-1}$ types of such Hopf algebras. If $b<\frac{n}{2}$, then $h i \equiv h^{\prime} i\left(\bmod p^{n-1}\right)$ is equivalent to $p^{n-2 b} \mid \alpha-\beta$, which means that for a fixed $h$ there are precisely $\left(p^{n-1-b}\right) /\left(p^{n-2 b}\right)=p^{b-1}$ elements $h^{\prime}$ for which $h^{\prime} i \equiv h i(\bmod$ $\left.p^{n-1}\right)$. This implies that there are $\left(p^{n-1-b}\right) /\left(p^{b-1}\right)=p^{n-2 b}$ elements $j$ such that $H(\lambda, i) \simeq H(\lambda, j)$. Therefore there exist $\left(p^{n-b}-p^{n-b-1}\right) /\left(p^{n-2 b}\right)=p^{b}-p^{b-1}$ types of such Hopf algebras. It follows that there are

$$
\sum_{1 \leq b \leq \frac{n}{2}}\left(p^{b}-p^{b-1}\right)+\sum_{\frac{n}{2}<b \leq n-1}\left(p^{n-b}-p^{n-b-1}\right)
$$

types of Hopf algebras of the form $H(\lambda, i)$. Adding the $p-1$ types of the form $H(\lambda)$ (see Lemma 6), an easy computation proves the required formula.

Remark 8. Note that Corollary 3 also follows from $[5,1.1 .1]$ for $\operatorname{dim} H=p^{2}, p$ prime. Contrary to the assertion in $[5,1.1 .1]$, if $N$ is not prime, then Hopf algebras such as $H(\lambda)$ appear. (An addendum to [5] will appear.)

Added in proof. The fact that pointed nonsemisimple Hopf algebras of dimension $p^{2}$ are Taft Hopf algebras seems to have been known for some time. Proofs appear also in [1], where Nichols is quoted, and in [16] where Andruwskiewitsch and Chin are acknowledged.

\section{REFERENCES}

[1] N. Andruskiewitsch and H.-J. Schneider, Hopf algebras of order $p^{2}$ and braided Hopf algebras of order $p$, J. Algebra 199 (1998), 430-454. CMP 98:06

[2] M. Beattie, S. Dăscălescu, L. Grünenfelder, C. Năstăsescu, Finiteness conditions, coFrobenius Hopf algebras and quantum groups, J. Algebra 200 (1998), 312-333. CMP 98:08

[3] M. Beattie, S. Dăscălescu, L. Grünenfelder, Constructing pointed Hopf algebras by Ore extensions, preprint.

[4] S. Gelaki, Quantum groups of dimension $p q^{2}$, Israel J. Math. 102 (1997), 227-267. CMP 98:06

[5] S. Gelaki, On Pointed Ribbon Hopf Algebras, J. Algebra 181 (1996), 760-786. MR 97d:16044

[6] C. Kassel, Quantum Groups, Graduate Texts in Mathematics 155 (1995), Springer Verlag. MR 96e: 17041

[7] R. Larson and D. Radford, Semisimple Hopf algebras, J. Algebra 171 (1995), 5-35. MR 96a: 16040

[8] A. Masuoka, Semisimple Hopf algebras of dimension 6,8, Israel J. Math. 92 (1995), 361-373. MR 96j:16045

[9] A. Masuoka, Self-dual Hopf algebras of dimension $p^{3}$ obtained by extension, J. Algebra 178 (1995), 791-806. MR 96j:16046

[10] A. Masuoka, The $p^{n}$ theorem for semisimple Hopf algebras, Proc. Amer. Math. Soc. 124 (1996), 735-737. MR 96f:16046

[11] A. Masuoka, Semisimple Hopf algebras of dimension 2p, Comm. Algebra 23 (1995), 1931-1940. MR 96e: 16050

[12] S. Montgomery, Hopf algebras and their actions on rings, CBMS no. 82, Amer. Math. Soc., 1993. MR 94i: 16019

[13] D. E. Radford, Operators on Hopf algebras, Amer. J. Math. 99 (1977), 139-158. MR 55:10505

[14] D. E. Radford, Irreducible representations of $\mathcal{U}_{q}(g)$ arising from $\operatorname{Mod}_{C^{1 / 2}}{ }$, Israel Math. Conference Proceedings 7 (1993), 143-170. MR 95b:17020 
[15] D. E. Radford, On Kauffman's knot invariants arising from finite-dimensional Hopf algebras, in "Advances in Hopf Algebras", Lecture Notes in Pure and Appl. Math., vol. 158, 205-266, Marcel Dekker, New York, 1994. MR 96g:57013

[16] D. Stefan, Hopf subalgebras of pointed Hopf algebras and applications, Proc. Amer. Math. Soc. 125 (1997), 3191-3193. MR 97m:16076

[17] Y. Zhu, Hopf algebras of prime dimension, Int. Math. Research Notices 1 (1994), 53-59. MR 94j:16072

Department of Mathematics and Computer Science, Mount Allison University, Sackville, New Brunswick, Canada E4L 1E6

E-mail address: mbeattie@mta.ca

Faculty of Mathematics, University of Bucharest, Str. Academiei 14, RO-70109 BuChaREST 1, Romania

E-mail address: sdascal@al.math.unibuc.ro

Department of Mathematics, Statistics and Computing Science, Dalhousie UniverSity, Halifax, Nova Scotia, Canada B3H 3J5

E-mail address: Luzius@mscs.dal.ca 